\title{
DETERMINATION OF THE RELATIVE CONCENTRATIONS OF RARE EARTH IONS BY X-RAY ABSORPTION SPECTROSCOPY: APPLICATION TO TERBIUM MIXED OXIDES
}

\author{
G. VAN DER LAAN \\ Department of Physical Chemistry, Materials Science Centre, University of Groningen, Nijenborgh 16, \\ 9747 AG GRONINGEN, The Netherlands \\ J. C. FUGGLE \\ Laboratory for Molecular Spectroscopy, Research Institute for Materials, Catholic University \\ of Nijmegen, Toernooiveld, 6525 ED NIJMEGEN, The Netherlands \\ M. P. VAN DIJK, A. J. BURGGRAAF \\ Department of Chemical Technology, Laboratory for Inorganic Materials Science, Twente University of \\ Technology, P.O. Box 217, 7500 AE ENSCHEDE, The Netherlands \\ and \\ J.-M. ESTEVA AND R. KARNATAK \\ Laboratoire pour l'Utilization du Rayonnement Electromagnétique, Bâtiment $209 \mathrm{c}$, Université \\ de Paris-Sud, 91405 ORSAY CEDEX, France
}

(Received 15 October 1985; accepted 7 November 1985)

\begin{abstract}
A method, based on X-ray absorption spectroscopy (XAS) in the range $0.8-1.5 \mathrm{keV}$, to determine the relative amounts of rare earth ions in different valencies is explained and tested for the case of terbium mixed oxides. The results are in agreement with those obtained by existing analytical methods. The XAS method is advantageous in that it can be applied where other, conventional, methods break down.
\end{abstract}

Keywords: X-ray absorption, rare earths, oxides, terbium, ionic conductivity, mixed valence.

\section{INTRODUCTION}

In this paper we show that, making use of the recently calculated spectra for the rare earth configurations and their tabulated properties by Thole [1], one can obtain information about the valency concentrations of rare earth ions in solid materials. These numbers are of technological importance and we will pick out an example.

Materials with conducting oxygen ions are frequently used in applications such as oxygen sensors, pumps and fuel cells in conjunction with platinum electrodes. It has been suggested that the electronic contribution to the conductivity of the solid electrolyte may improve the performance of the noble metal electrode or can even substitute these [2-5]. This has led to extensive studies of mixed oxides, like $\left(\mathrm{Tb}_{x} \mathrm{Gd}_{1-x}\right)_{2} \mathrm{Zr}_{2} \mathrm{O}_{7+y}$ in which both $\mathrm{Tb}^{3+}$ and $\mathrm{Tb}^{4+}$ are found. From studies of electrical conductivity and ionic transport properties of these materials it is apparent that there is an electronic contribution to the conductivity due to hopping of electrons from $\mathrm{Tb}^{3+}$ to $\mathrm{Tb}^{4+}$ sites [6, 7]. The activation energy for this latter process is thought to arise from the lattice distortion around the $\mathrm{Tb}^{3+}$ and $\mathrm{Tb}^{4+}$ ions and can be treated in small polaron models [79]. In order to put the understanding of such electronic conductivity on a firm basis, it is necessary to know the relative quantities of reduced and oxidized forms of the ions in the lattices. There are not many methods for making this analysis. In some cases this is possible using thermogravimetric analysis (TGA). In this method the oxygen weight loss as a function of temperature or oxygen partial pressure is measured and if the stoichiometry of the sample is known at one temperature and pressure, then the stoichiometry at all temperatures and pressures can be deduced. This approach is not possible if one of the components of the mixed oxide is volatile or if more than one of the atoms present can exist in multiple valencies. Other possible methods, such as X-ray photoelectron spectroscopy, are very surface sensitive and may suffer from surface decomposition in the ultra-high vacuum environments used.

In view of this paucity of methods to analyse the valency of metal ions in mixed oxide conductors we have attempted to use X-ray absorption spectroscopy (XAS) at the $3 d$ edges of the rare earths. These spectra are known to have sharp peaks and multiplets which are characteristic of the metal ion valency [1]. We will show that the XAS method does yield semi-quantitative results for the rare earth valencies in these materials. Another example of this method for the case of mixed Ce valencies can be found in Ref. [10].

\section{EXPERIMENTAL METHODS}

All materials were prepared by means of thermolysis of a viscous solution of citrate complexes of the con- 
stituent metal ions. After calcination at $800^{\circ} \mathrm{C}$ for 6 hours the powders were pressed isostatically and sintered into dense ceramics at a temperature between 1200 and $1550^{\circ} \mathrm{C}$. X-ray diffraction revealed the fluorite structure for all materials investigated.

To study the stoichiometry of these compounds thermogravimetric analysis (TGA) runs were carried out for several compositions in the solid solution series $\left(\mathrm{Tb}_{x} \mathrm{Gd}_{1-x}\right)_{2} \mathrm{Zr}_{2} \mathrm{O}_{7+y}$. Extensive results of TGA measurements together with results of electrical conductivity measurements have been reported elsewhere [7]. In summary, runs were carried out in air and under reducing conditions in the temperature range 300 $1000^{\circ} \mathrm{C}$. The latter conditions were realized with a gas mixture consisting of $1 \% \mathrm{CO}$ and $99 \% \mathrm{CO}_{2}$, resulting in a sample stoichiometry for the $\left(\mathrm{Tb}_{x} \mathrm{Gd}_{1-x}\right)_{2} \mathrm{Zr}_{2} \mathrm{O}_{7+y}$ compositions with all $\mathrm{Tb}$ in the $3+$ state [7]. This was used as a starting point to determine the stoichiometry at all temperatures in air.

For XAS experiments discs of the ceramic specimen were equilibrated at $600^{\circ} \mathrm{C}$ in air and quenched to room temperature rapidly. Reduced samples were obtained as above for the TGA measurements.

$\mathrm{X}$-ray absorption spectra of the $\mathrm{Tb} \mathrm{M}_{4,5}$ cxcitations were obtained using the synchrotron radiation emitted by the Anneau de Collisions d'Orsay (ACO) at Orsay. The use of a constant deviation double crystal monochromator equipped with beryl crystals results in an energy resolution of $0.4 \mathrm{eV}$ [11]. The spectra were recorded by the electron yield method so that no samples of thin foils were required. Checks were made on the $X$-ray flux using an Al sample, displaying a flat $X$-ray flux in the energy region of the $\mathrm{Tb} \mathrm{M}_{4,5}$ absorptions. The energy scale was calibrated by setting the La $3 d_{3 / 2}$ and $\mathrm{Mg} 1 s$ edges to an energy of respectively 850.5 and $1303.0 \mathrm{eV}$.

The samples were measured under ultra high vacuum conditions. The spectra were identical for a sample without any surface treatment and for scraping the surface in the vacuum, indicating that bulk and surface have the same composition.

\section{RESULTS}

Fig. 1 shows the measured XAS spectrum from $\mathrm{Tb}^{3+}$ in $\mathrm{TbAl}_{2}$ compared with the calculated $\mathrm{XAS}$ spectra from $\mathrm{Tb}^{3+}$ and $\mathrm{Tb}^{4+}$. The spectra have been explained as being transitions from the Hund's rule ground state to final states where a $3 d$ core electron has been excited to the $4 f$ level: $3 d^{10} f^{8}\left({ }^{7} \mathrm{~F}_{6}\right) \rightarrow 3 d^{9} 4 f^{9}$ and $3 d^{10} f^{7}\left({ }^{8} \mathrm{~S}_{12}\right)-3 d^{9} 4 f^{8}$ for the $\mathrm{Tb}^{3+}$ and $\mathrm{Tb}^{4+}$, respectively. The final states are multiplet split but the dipole selection rules severely limit the number of accessible states [1]. The spectra are roughly separated in a $3 d_{5 / 2}\left(\mathrm{M}_{5}\right)$ and a $3 d_{3 / 2}\left(\mathrm{M}_{4}\right)$ multiplet at $\sim 1240$ and $\sim 1270 \mathrm{eV}$ respectively, due to the large $3 d$ spinorbit splitting. It is clear that the $\mathrm{Tb}^{3+}$ and $\mathrm{Tb}^{4+}$ spectra are quite distinct, but that they will overlap when the two species are present together. We use the $M_{5}$ peaks for analysis because they are sharper, stronger, and less influenced by Fano effects [1].

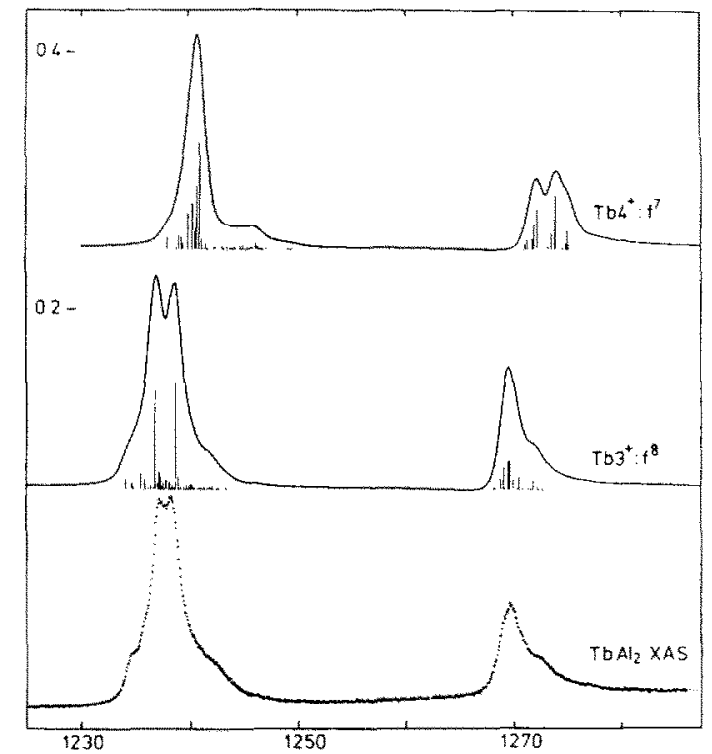

Fig. 1. Experimental $\mathrm{M}_{4,5} \mathrm{X}$-ray absorption spectrum of $\mathrm{TbAl}_{2}$ compared with the calculated spectra for $\mathrm{Tb}^{3+}$ and $\mathrm{Tb}^{4+}$.

Fig. 2 shows the XAS spectra of various Tb mixed oxide samples. It is seen that the spectrum of the reduced mixed oxide of $\mathrm{Tb}$ is very similar to the calculated $\mathrm{Tb}^{3+}$ ion spectrum and $\mathrm{Tb}$ in $\mathrm{TbAl}_{2}$ (Fig. 1). In this spectrum we should surely have detected a $5 \%$ contribution from $\mathrm{Tb}^{4+}$ but none was found, in agreement with the conclusions of thermogravimetric analysis [7].

If we now look at the oxidized forms of $\mathrm{Tb}_{1.2} \mathrm{Gd}_{0.8} \mathrm{Zr}_{2} \mathrm{O}_{7}$ and $\mathrm{Ce}_{0.7} \mathrm{~Tb}_{0.3} \mathrm{O}_{1.85+y}$ we see distinct shoulders on the high energy side of the main peaks. These are attributed to the $\mathrm{Tb}^{4+}$ contributions in the spectra.

In order to extract the amount of $\mathrm{Tb}^{3+}$ and $\mathrm{Tb}^{4+}$ from the experimental spectra, one could deconvolute the theoretical spectra. However, there are minor differences between theory and experiment, partly due to the fact that only atomic effects and no solid state effects have been taken into account in the calculated spectra. In order to avoid possible complications we simply use the experimental $\mathrm{Tb}^{3+}$ curve from the reduced compound and substract it from the total spectra of the oxides. This procedure is demonstrated in Fig. 2. We already have seen from Fig. 1 that at the relative maximum at the left side in the $\mathrm{Tb}^{3+}$ peak $(\sim 1238 \mathrm{eV})$, hardly any $\mathrm{Tb}^{4+}$ contribution is present. Thus we can use this point to normalize the spectra. The dashed line in Figs. 2(a) and (b) is the $\mathrm{Tb}^{3+}$ contribution whereas the full line represents the $\mathrm{Tb}^{4+}$ contribution.

There is a small background problem in some of the GdTbZr oxides (as seen in Fig. 2a), which may be due to the slowly varying EXAFS oscillations from the nearby $\mathrm{Gd} \mathrm{M}_{4,5}$ excitations at about 23 and $53 \mathrm{eV}$ lower excitation energy. This complication is not present in the $\mathrm{Ce}-\mathrm{Tb}$ oxides where the $\mathrm{Ce} \mathbf{M}_{4,5}$ transition is at much lower excitation energy. Choice of the back- 


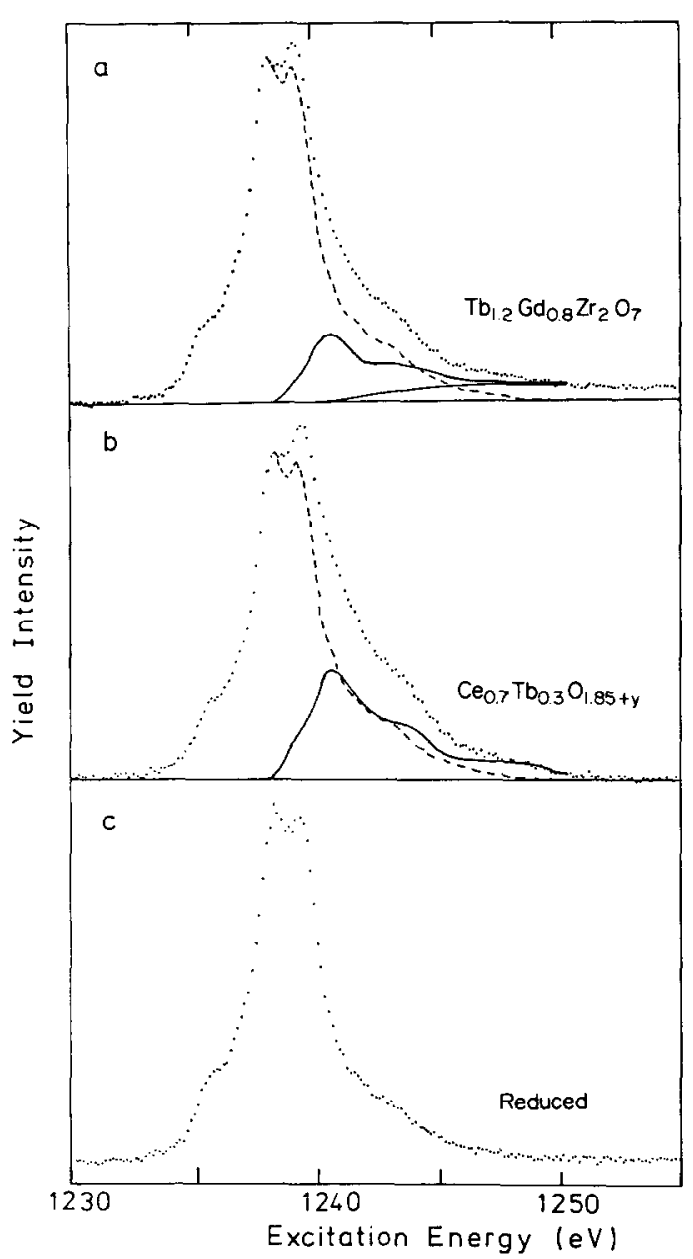

Fig. 2. The experimental $\mathrm{M}_{4,5}$ spectra from $\mathrm{Tb}_{1.2} \mathrm{Gd}_{0.8} \mathrm{Zr}_{2} \mathrm{O}_{7+y}$ (a), from $\mathrm{Ce}_{0.7} \mathrm{~Tb}_{0.3} \mathrm{O}_{1.85+y}$ (b) and from material a in reduced form (c) are given by the dotted curves. The $\mathrm{Tb}^{3+}$ and $\mathrm{Tb}^{4+}$ contributions in the oxides are given by the dashed line and the solid line, respectively: also given is a suggested background (lowest solid line).

ground is somewhat arbitrary and is certainly a limitation, although not a dramatic one. Note that the position of the $\mathrm{Tb}^{4+}$ contribution is within $0.2 \mathrm{eV}$ of that predicted [1].

The relative valency concentrations are obtained by dividing the areas of the $\mathrm{Tb}^{3+}$ and $\mathrm{Tb}^{4+}$ contributions by the theoretical $\mathrm{M}_{5}$ cross sections. Theoretical cross sections for all rare earths are found in Thole's tables [1]. The XAS values obtained for the $\mathrm{Tb}^{4+}$ and $\mathrm{Tb}^{3+}$ percentages are given in Table 1. Also presented are the percentages for other $\mathrm{Tb}$ mixed oxides studied, $\mathrm{Tb}_{0.8} \mathrm{Gd}_{1.2} \mathrm{Zr}_{2} \mathrm{O}_{7+y}$ and $\mathrm{Tb}_{0.6} \mathrm{Bi}_{1.4} \mathrm{O}_{3+y}$. The values are comparable to values from TGA. Note that the independent determination of the amount of $\mathrm{Tb}^{4+}$ using TGA measurements is almost impossible in the Ce$\mathrm{Tb}$ oxide, due to the presence of both $\mathrm{Ce}$ and $\mathrm{Tb}$ in two valencies.

\section{DISCUSSION}

The ability to determine by XAS the $\mathrm{Tb}^{3+}$ and $\mathrm{Tb}^{4+}$ quantities in the mixed oxides depends on the sepa- ration of the $\mathrm{Tb}^{3+}$ and $\mathrm{Tb}^{4+}$ contributions to the spectrum. There are three tests for the validity of this separation.

1) Is the $\mathrm{Tb}^{4+}$ contribution at the predicted energy? Here the answer is yes within $0.2 \mathrm{eV}$.

2) Is the shape of the residue attributed to $\mathrm{Tb}^{4+}$ reasonable? Here we find a larger width to the $\mathrm{Tb}^{4+}$ contribution than calculated. There are various possible explanations for this but most probably it is a result of small changes in the shape of the $\mathrm{Tb}^{3+}$ contribution from one compound to another.

3) Is the $\mathrm{Tb}^{4+}$ weight derived from these measurements comparable to that from the standard methods? The standard method for these materials is thermogravimetric analysis and we have compared various results from TGA with our XAS data in Table 1. The agreement is remarkably good.

The accuracy of XAS measurements of the valency of $\mathrm{Tb}$ is difficult to assess. Systematic errors can enter due to the background subtraction or small changes in the $\mathrm{Tb}^{3+}$ line shape. We estimate an accuracy of $\sim 3-5 \%$ in the $\mathrm{Tb}^{4+}$ content.

The sample of $\mathrm{Ce}_{0.7} \mathrm{~Tb}_{0.3} \mathrm{O}_{1.85+y}$ showed a very large $\mathrm{Tb}^{4+}$ contribution of $\sim 25 \%$, which was much higher than for the TbGdZr mixed oxides. This is in accord with preliminary conductivity measurements $[6,12]$. We also find a very low $\mathrm{Tb}^{4+}$ concentration in the $\mathrm{Tb}_{0.6} \mathrm{Bi}_{1.4} \mathrm{O}_{3+y}$ sample exposed to the same oxygen treatment. Clearly the lattice has a significant effect on the equilibrium $\mathrm{Tb}^{3+}: \mathrm{Tb}^{4+}$ ratios. Considering effects of strain energy, one might expect larger fractions of $\mathrm{Tb}^{3+}$ to be present in the host lattice with the larger cations, since the ionic radius of $\mathrm{Tb}^{3+}(1.04 \AA$, Shannon and Prewitt values for 8-coordination [13]) is larger than the ionic radius of $\mathrm{Tb}^{4+}(0.88 \AA)$. Thus the $\mathrm{Tb}$ ions fit better in their host lattice and the lattice has a lower free energy. This concept explains the result obtained for $\mathrm{Ce}_{0.7} \mathrm{~Tb}_{0.3} \mathrm{O}_{1.85+y}$ compared to the result obtained for $\mathrm{Tb}_{0.6} \mathrm{Bi}_{1.4} \mathrm{O}_{3+y}$. Extending this concept to $\mathrm{TbGdZr}$ mixed oxides, the results indicate that the $\mathrm{Tb}$ ions occupy Gd ion lattice sites, which are nonequivalent to the $\mathrm{Zr}$ lattice sites. This is in accord with the observations of short range order in the fluorite lattice and the formation of pyrochlore ordered microdomains for the TbGdZr mixed oxides [6, 14].

\section{CONCLUSIONS}

We have shown that X-ray absorption is a suitable quantitative tool for analysis of the valency of $\mathrm{Tb}$ in

Table 1. \% of $\mathrm{Tb}^{4+}$ as obtained from XAS and TGA measurements

\begin{tabular}{lcc}
\hline & XAS & TGA \\
\hline $\mathrm{Tb}_{1.2} \mathrm{Gd}_{0.8} \mathrm{Zr}_{2} \mathrm{O}_{7+y}$ & 15 & 14 \\
$\mathrm{~Tb}_{0.8} \mathrm{Gd}_{1.2} \mathrm{Zr}_{2} \mathrm{O}_{7+y}$ & 13 & 14 \\
$\mathrm{Ce}_{0.7} \mathrm{~Tb}_{0.3} \mathrm{O}_{1.85+y}$ & 25 & - \\
$\mathrm{Tb}_{0.6} \mathrm{Bi}_{1.4} \mathrm{O}_{3+y}$ & 5 & - \\
$\mathrm{Tb}_{0.8} \mathrm{Gd}_{1.2} \mathrm{Zr}_{2} \mathrm{O}_{7+y}$ (reduced) & 0 & 0 \\
\hline
\end{tabular}


mixed oxide materials. In some cases the concentration of $\mathrm{Tb}$ was as low as $\sim 5$ atomic\% $\mathrm{Tb}$, but we still obtain usable results with recording times as low as ten minutes. Thus the sensilivity of XAS is adjudged to be high. Also for the $\mathrm{Ce}_{0.7} \mathrm{~Tb}_{0.3} \mathrm{O}_{1.85+y}$ and $\mathrm{Tb}_{0.6} \mathrm{Bi}_{1.4} \mathrm{O}_{3+y}$ the standard method, thermogravimetric analysis, is not applicable. We thus conclude that XAS is a suitable adjunct to the standard analytical methods for study of such systems.

Acknowledgements-We are grateful to the LURE technical staff for their valuable aid. We also thank G. A. Sawatzky and B. T. Thole for valuable discussions and Miss M. van Hemert for the preparation of the $\mathrm{Tb}_{0.6} \mathrm{Bi}_{1.4} \mathrm{O}_{3+y}$ specimen. This work was supported by the Netherlands Foundation for Chemical Research (SON) with financial aid from the Netherlands Organization for the Advancement of Pure Research (ZWO) and by the Centre Nationale de la Recherche Scientifique (CNRS) in France.

\section{REFERENCES}

1. Thole B. T., van der Laan G., Fuggle J. C., Sawatzky G. A., Karnatak R. C. and Esteva J. M., Phys. Rev. B32, 5107 (1985).
2. Schouler E. J L Solid State Ionics $9 / 10,945$ (1983)

3. Badwal S. P. S., J. Electroanal. Chem. 46, 425 (1983).

4. Van Dijk M. P., ter Maat J. H. H., Roelofs G., Bosch H. van de Velde G. M. H., Gellings P. J. and Burggraaf A. J., Mat. Res. Bull. 19, 1149 (1984).

5. Takasu Y., Sugion T. and Matsuda Y., J. Appl. Electrochem. 14, 79 (1984).

6. Van Dijk M. P., Ph.D. Thesis, Ordering electrical conductivity and electrode properties of ceramics with fluorite related structures. T. H. Twente, The Netherlands (1985).

7. Van Dijk M. P., de Vries K. J. and Burggraaf A. J., Solid State Ionics 16, 211 (1985).

8. Austin I. G. and Mott N. F., Adv. Phys. 18, 41 (1969)

9. Tuller H. L. and Nowick A. S., J. Phys. Chem. Solids 38, 859 (1977).

10. Fransen T., Gellings P. J., Fuggle J. C., van der Laan G., Esteva J. M. and Karnatak R. C., Appl. Surface Sci. 20, 257 (1985).

11. Lemonnier M., Collet O., Depautex C., Esteva J. M. and Raoux D., Nucl. Instr. Mcth. 152, 109 (1978).

12. Burggraaf A. J., van Dijk M. P. and de Vries K. J., to be published in Solid State Ionics (Special Issue: Proceedings Sixth International Conference on Solid State Ionics), Lake Tahoe, Nevada (1985).

13. Shannon R. D. and Prewitt C. T., Acta Cryst. B25, 925 (1969).

14. Van Dijk M. P., Mijlhoff F. C. and Burggraaf A. J., J. Solid State Chemistry, 1986, in press. 